\title{
ViSUALYRE: Multimodal visualization of lyrics
}

\author{
Gamar Azuaje \\ Kongmeng Liew \\ \{azuaje_suarez.gamar_ivan.ac4,liew.kongmeng\} \\ @is.naist.jp \\ Nara Institute of Science and \\ Technology \\ Ikoma, Nara, Japan
}

\author{
Elena V. Epure \\ Deezer Research \\ Paris, France \\ eepure@deezer.com
}

\author{
Shuntaro Yada \\ Shoko Wakamiya \\ Eiji Aramaki \\ \{s-yada,wakamiya,aramaki\} \\ @is.naist.jp \\ Nara Institute of Science and \\ Technology \\ Ikoma, Nara, Japan
}

\begin{abstract}
In this paper, we present Visualyre, a web application that synthesizes images based on the semantics of the lyrics, and the mood of the music. We use a multimodal approach, generating initial images with a text-to-image generative models from the lyrics (text) of a song, followed by a style transfer model conditioned to the mood of the music (audio). Our target user base is the independent music artist community, by providing a means for composers and songwriters to generate suitable images for their music (such as album covers). We discuss the possible usage of such application, as well as the possible improvements in future iterations.
\end{abstract}

\section{CCS CONCEPTS}

- Human-centered computing $\rightarrow$ Graphical user interfaces; Webbased interaction; - Computing methodologies $\rightarrow$ Neural networks; • Applied computing $\rightarrow$ Sound and music computing.

\section{KEYWORDS}

deep learning, image generation, style transfer, lyrics, mood detection

\section{ACM Reference Format:}

Gamar Azuaje, Kongmeng Liew, Elena V. Epure, Shuntaro Yada, Shoko Wakamiya, and Eiji Aramaki. 2021. VIsUALYRE: Multimodal visualization of lyrics. In Proceedings of the 16th International Audio Mostly Conference (AM'21). ACM, New York, NY, USA, 5 pages. https://doi.org/10.1145/3478384. 3478420

\section{INTRODUCTION}

Since the development of Generative Adversarial Networks (GANs) in Computer Vision in 2014, deep learning-based image generation techniques have been increasingly popular [8]. In this paper, we propose one practical application of text-to-image GAN models: album art generation from music and lyrics. Album art has often accompanied commercial music as an accompanying abstract, yet crossmodally coherent visual representation [2]. However, such

Permission to make digital or hard copies of all or part of this work for personal or classroom use is granted without fee provided that copies are not made or distributed for profit or commercial advantage and that copies bear this notice and the full citation on the first page. Copyrights for components of this work owned by others than ACM must be honored. Abstracting with credit is permitted. To copy otherwise, or republish, to post on servers or to redistribute to lists, requires prior specific permission and/or a fee. Request permissions from permissions@acm.org.

AM'21, September 01-03, Trento, Italy

(C) 2021 Association for Computing Machinery.

ACM ISBN 978-1-4503-8569-5/21/09..\$15.00

https://doi.org/10.1145/3478384.3478420 artwork may not be easily obtainable, especially for independent musicians who lack the financial means or artistic resources to create it themselves. As such, we propose that GANs, despite their limits on semantic representation, may provide a possible solution. By generating images based on the lyrics of a given song, GANs can capture some extent of the semantic relationships that exists in a particular song.

GAN models are composed of two neural networks, a generator that tries to replicate real images from a dataset, and a discriminator that distinguishes real images from replicates. The training of the model converges when the the discriminator is unable to distinguish real images from replicates created by the generator.

One particularly interesting application of these networks has been in the area of visual art, for example, Elgammal et al. [4] utilized GANs to generate art based on the Wikiart dataset, spanning paintings from the 1400 s to the 2000s. Participants in the followup experiment rated the generated images as more inspiring, and aesthetically pleasing than (human-painted) contemporary art.

However, GAN-synthesised images are often visually indeterminate [9]; for our goal of generating album art, they may not be good enough to adequately represent the meanings or emotions conveyed on a given song. As such, we also propose the addition of a second module in our architecture, the usage of audio analysis to determine the mood of a song to consequently fine-tune the synthesized images using Style Transfer [6]. Style Transfer is a deep-learning based method that combines the neural representations of two images, resulting in a third image that is a blended mix of the two constituent images. We first collect a set of 'emotional' images that correspond to each different mood categories classified by the audio analysis models. Next, based on the mood classification of the song, we merge the GAN-synthesized image with a corresponding 'emotional' image through style transfer. We think that by employing a multimodal approach that uses both lyrics and audio is a better way to create images that are close to the vision that the author had in mind for an album art, than generating images based solely on the lyrics as we are augmenting the images with additional information not present in the lyrics.

In sum, we develop Visualyre, a web application that generates images for musicians to use as an album art. Our proposed model architecture for Visualyre consists of three core modules used in sequence. First, a GAN synthesizes images based on the lyrics (lyrical) of a song. Followed by an audio analyzer that classifies the mood of the song from audio features. Finally, a style-transfer module augments these images by combining them with priorly selected 
images that matches the (musical) mood of the song. We hope that this provides a reliable means for musicians to generate album art, particularly for musicians who may not have the means to obtain one otherwise.

\section{RELATED WORK}

There has been previous interest in methods of visualizing music. One approach is the visualization through musical moods. Laurier et al. [12] designed an application that visualizes moods probabilities in real-time, displaying the probability of each mood during a specific time frame using bar charts. Husain et al. [11] followed the same approach, designing visual textures based on the relationship between moods and different visual elements, such as color and shapes.

Another approach is the visualization from lyrics. Funasawa et al. [5] created a system to identify keywords within each line, and used image retrieval to form a slideshow that displays images corresponding to words in each line of lyrics.

Visualyre adopts both approaches in a multimodal fashion. The lyrics are visualized line-by-line, but rather than using keywords to retrieve existing images, it uses image generation to synthesize new images using each sentence as an input. The moods extracted from the audio are visualized via Style Transfer, using pictures related to each mood as texture images, generating images suitable for use as album art.

\section{SYSTEM DESCRIPTION}

Visualyre aims to support independent musicians by generating an album artwork according to the semantics and emotional moods of the input song. Particularly, this system uses the lyrics (text) and music (audio) of the song as features for the generation of the image. To fine-tune and adjust the generated images, Visualyre employs Style Transfer, achieving a similar effect to image filters available in smartphone camera applications.

Multiple modules are needed to create representative images of a particular pair of audio files and lyrics. The modules of Visualyre include a text-to-image generation model to synthesize images from lyrics, an audio analyzer to evaluate the moods of a song file from its audio features, and a style transfer model to adapt an image to a particular mood. The system architecture is shown in Figure 1.

\subsection{Image generation}

Images generated by a text-based image generation model are conditioned to features extracted from an input sentence with a textencoder. Multiple approaches have been proposed over the years, such as using a stacked approach to refine the images [19], employing attention to synthesize fine-grained details [18], and inferring semantic information to guide the generation [10].

DM-GAN [20] makes use of dynamic memory to refine the content of the synthesized images, even if the quality of the previous images is not good. This generative mobile is trained using the MS COCO Dataset [15], which contains a total of 123,287 images (82,783 for training and 40,504 for validation) with five captions per image.

Visualyre uses DM-GAN to visualize the lyrics, generating an image for each sentence in the input lyrics.

\subsection{Audio Analysis}

Listening to music encompasses perceiving emotionality [13]. Therefore, it is essential to understand the emotions portrayed by the artist in their songs in order to generate a representative album art.

We utilize binary classifiers for four different emotions to capture the mood present in musical pieces. These classifiers are trained to detect anger, happiness, sadness, and relaxation by using the musical features extracted from the audio signal of a song. They were introduced by Laurier et al, [16] initially using Support Vector Machines, and were recently ported to deep convolutional neural networks by the Music Technology Group [1].

For our model, we normalized the positive label of these classifiers and applied them towards the enhancement of the generated images (see section on Style Transfer), to give them an artistic feel.

\subsection{Style Transfer}

Style transfer is the process of changing the style of an image to match the style of another image, while still preserving its original content [17]. In previous research, models were restricted to only change the style of a specific image, as these models had a different trained model for each style image $[6,14]$. Ghaidi et al. [7] introduced a style prediction network that can predict the style embeddings from an arbitrary style image, effectively enabling stylization using any pair of content and style images. Their network is trained using content images from the ImageNet ${ }^{1}$ dataset and the Kaggle Painter By Numbers $(\mathrm{PBN})^{2}$, a dataset consisting of 79,433 paintings.

3.3.1 Style Sampling. For Visualyre, we prepared a small dataset, Style Bank: A collection of style images based on the four different moods of the binary classifiers used in the audio analysis (anger, happiness, sadness, and relaxation). We chose images from Unsplash $^{3}$ by using search queries related to the target four moods, such as 'fury,' 'joy,' 'calm,' and 'sorrow.' Our intention is to use styletransfer to combine these 'mood' images with the images generated by the GAN model. To determine which 'mood' image should be combined with the GAN generated image, we rely on the mood classification scores from the audio analysis. We first normalized these scores computed at an earlier step, to obtain an audio-based Mood Probability, assigning a probability to each mood based on the score of the classifiers. The higher the score the most likely that a mood will be sampled for style transfer. After selecting a mood, we select an image representative of the chosen mood as the style image from the Style Bank.

We sample eight different style images, applying Style Transfer to a synthesized image previously selected by the user. Together with the original synthesized image we show the user a total of nine different images to choose from.

\subsection{Graphical User Interface}

Visualyre synthesizes an album art in a sequential manner, involving the usage of different modules. To make this process as seamless and dynamic as possible for the end user, we opted for a responsive

\footnotetext{
${ }^{1}$ https://www.image-net.org/

${ }^{2}$ https://www.kaggle.com/c/painter-by-numbers

${ }^{3}$ https://unsplash.com/
} 


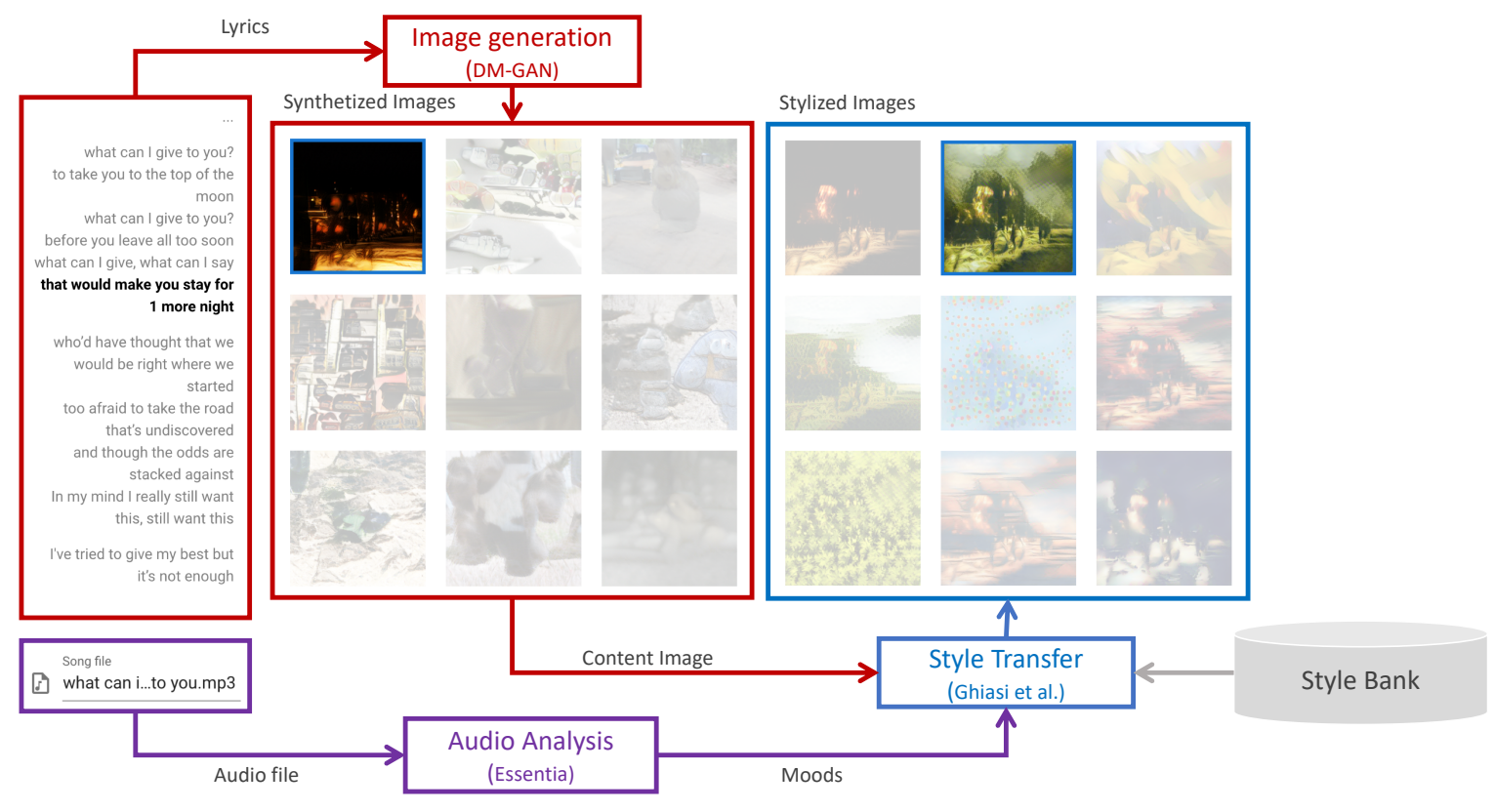

Figure 1: System Architecture: Lyrics are used to synthesize images with a text-based image generator. The audio file is analyzed to evaluate the presence of four different moods. This evaluation is used to sample which style images are used in Style Transfer.

layout and a minimalist design, showing the components only as they are needed. Figure 2 shows the graphical user interface of Visualyre and its different components.

- Song input: a form where users add lyrics and upload an audio file.

- Navigation menu: buttons that enable travel to the next step or to the previous one. An additional button indicates if the system has finished analyzing the moods from the submitted audio file.

- Progress tracker: allows users to check their current step at any time. The name of each step provides a subtle description on the desired action during a specific step.

- Image selection: a grid where users can select or hover over generated or stylized images. Multiple images are displayed simultaneously, using pagination to display further images when necessary. To advance to the style selection, a user must select a synthesized image. Similarly, a selection of a stylized image is required to download an image.

- Lyrics display: a container where users to view the input lyrics during the selection of synthesized images. When an image is hovered or selected, the system displays a fragment of the lyrics, highlighting the sentence used to generate each synthesized image.

It is worth noting that the entire process occurs on the same page without reloading or redirecting to another URL. The UI components and their respective content change reactively according to the user's interaction with the Visualyre.

\section{SYSTEM INTERACTION}

The procedure of how to use Visualyre from start to finish is described as follows:

(1) The user clicks START HERE on the home screen and is redirected to the song input form.

(2) The user inputs the lyrics in the text field and uploads an audio file. The CONTINUE button is then enabled.

(3) The user clicks CONTINUE and is redirected to the image selection. In the background, the generator starts generating images from the lyrics while the audio analyzer starts to evaluate the moods from the audio file.

(4) The images are generated and they are shown in a grid-like fashion.

(5) The audio analyzer finishes evaluating the moods from the input file. The top left button in the navigation changes to READY indicating that the mood detection of the audio file has finished.

(6) The user selects a synthesized image. The mood detection has already finished and the CONTINUE button is enabled.

(7) The user clicks CONTINUE and is redirected to the style selection.

(8) Style transfer is applied to the synthesized image by sampling style images conditioned to the results of the mood detection from the audio file.

(9) The stylized images are created and they are shown in a grid-like fashion. 


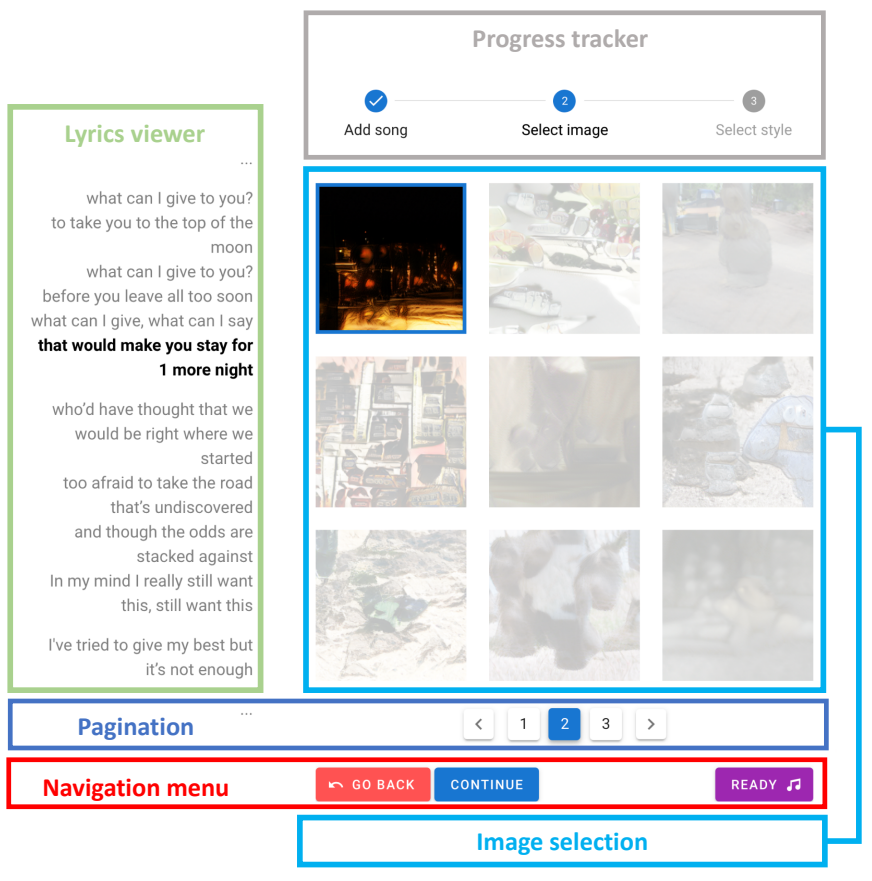

Figure 2: Graphical User Interface of Visualyre and its components. This figure shows the selection of a synthesised image from the image generator prior to applying style transfer.

(10) The user selects an stylized image, the image is shown on the left with a DOWNLOAD button.

(11) The user proceeds click DOWNLOAD, acquiring their desired stylized image.

\section{DISCUSSION AND FUTURE DIRECTIONS}

In this paper, we propose a novel application of text-to-image computational techniques in the form of Visualyre, an approach for lyric visualization that combines two modules (text-based image generation via DM-GAN and style transfer) to sequentially generate images based on lyric segments and the overall mood of the songs. Combined with the in-built possibility for user selection and input, we think that this method allows for the generation of a final image that can capture some aspect of semantic meaning or mood from a song. However, more detailed user experience (UX) research is needed to quantitatively and qualitatively determine the usefulness of this application for the indie music community.

Moving forward, we consider some areas where Visualyre can be improved on. Firstly, our GAN module is currently generating images to every line in the lyrics. Some of these lines may be too short or contain only abstract concepts, which are very difficult for the image generation model to synthesize. As such, one improvement could be to generate images only when the line contains at least one word with a concrete meaning. Secondly, Visualyre only supports lyrics written in English. Another improvement is adding multilingual support for the application, perhaps by training GAN models with multilingual datasets, or by adding machine translation capabilities within Visualyre's architecture. Finally, we note that the downloadable images have low resolution. We plan to use Super Resolution models [3] to enable high-resolution download in future iterations.

With Visualyre, one of our goals is to bring a modest contribution to the independent music community, by generating artwork to match the musical and artistic intentions of the artists. In the future, we will explore other possible applications, such as allowing users to browse music through synthesized images.

\section{ACKNOWLEDGMENTS}

The authors would like to thank Marcus 李俊緯 for consenting to the use of his track 'what can I give to you' in the figures and the demonstration video.

\section{REFERENCES}

[1] Pablo Alonso-Jiménez, Dmitry Bogdanov, Jordi Pons, and Xavier Serra. 2020. Tensorflow audio models in essentia. In ICASSP 2020-2020 IEEE International Conference on Acoustics, Speech and Signal Processing (ICASSP). IEEE, 266-270.

[2] Robert J Belton. 2015. The Narrative Potential of Album Covers. Studies in Visual Arts and Communication: an international journal (2015), 1-7.

[3] Chao Dong, Chen Change Loy, Kaiming He, and Xiaoou Tang. 2015. Image super-resolution using deep convolutional networks. IEEE transactions on pattern analysis and machine intelligence 38, 2 (2015), 295-307.

[4] Ahmed Elgammal, Bingchen Liu, Mohamed Elhoseiny, and Marian Mazzone. 2017. CAN: Creative Adversarial Networks, Generating "Art" by Learning About Styles and Deviating from Style Norms. arXiv:1706.07068 [cs.AI]

[5] Shintaro Funasawa, Hiromi Ishizaki, Keiichiro Hoashi, Yasuhiro Takishima, and Jiro Katto. 2010. Automated Music Slideshow Generation Using Web Images Based on Lyrics.. In ISMIR. 63-68.

[6] Leon A. Gatys, Alexander S. Ecker, and Matthias Bethge. 2015. A Neural Algorithm of Artistic Style. CoRR abs/1508.06576 (2015). arXiv:1508.06576 http://arxiv.org/abs/1508.06576

[7] Golnaz Ghiasi, Honglak Lee, Manjunath Kudlur, Vincent Dumoulin, and Jonathon Shlens. 2017. Exploring the structure of a real-time, arbitrary neural artistic stylization network. arXiv preprint arXiv:1705.06830 (2017).

[8] Ian J. Goodfellow, Jean Pouget-Abadie, Mehdi Mirza, Bing Xu, David WardeFarley, Sherjil Ozair, Aaron Courville, and Yoshua Bengio. 2014. Generative Adversarial Nets. In Proceedings of the 27th International Conference on Neural Information Processing Systems - Volume 2 (Montreal, Canada) (NIPS'14). MIT Press, Cambridge, MA, USA, 2672-2680.

[9] Aaron Hertzmann. 2020. Visual Indeterminacy in GAN Art. In ACM SIGGRAPH 2020 Art Gallery (Virtual Event, USA) (SIGGRAPH '20). Association for Computing Machinery, New York, NY, USA, 424-428. https://doi.org/10.1145/3386567. 3388574

[10] Seunghoon Hong, Dingdong Yang, Jongwook Choi, and Honglak Lee. 2018. Inferring semantic layout for hierarchical text-to-image synthesis. In Proceedings of the IEEE Conference on Computer Vision and Pattern Recognition. 7986-7994.

[11] Adzira Husain, Mohd Fairuz Shiratuddin, and Wai Wong Kok. 2015. Establishing a framework for visualizing music mood using visual texture. (2015).

[12] Cyril Laurier and Perfecto Herrera. 2008. Mood cloud: A real-time music mood visualization tool. Proceedings of the Computer Music Modeling and Retrieval (2008).

[13] Cyril Laurier, Owen Meyers, Joan Serra, Martin Blech, and Perfecto Herrera. 2009. Music mood annotator design and integration. In 2009 Seventh International Workshop on Content-Based Multimedia Indexing. IEEE, 156-161.

[14] Chuan Li and Michael Wand. 2016. Precomputed real-time texture synthesis with markovian generative adversarial networks. In European conference on computer vision. Springer, 702-716.

[15] Tsung-Yi Lin, Michael Maire, Serge Belongie, James Hays, Pietro Perona, Deva Ramanan, Piotr Dollár, and C Lawrence Zitnick. 2014. Microsoft coco: Common objects in context. In European conference on computer vision. Springer, 740-755.

[16] Christine Mohn, Heike Argstatter, and Friedrich-Wilhelm Wilker. 2011. Perception of six basic emotions in music. Psychology of $\mathrm{Mu}$ sic 39, 4 (2011), 503-517. https://doi.org/10.1177/0305735610378183 arXiv:https://doi.org/10.1177/0305735610378183

[17] Xuexiang Xie, Feng Tian, and Hock Soon Seah. 2007. Feature guided texture synthesis (fgts) for artistic style transfer. In Proceedings of the 2nd international conference on Digital interactive media in entertainment and arts. 44-49. 
[18] Tao Xu, Pengchuan Zhang, Oiuyuan Huang, Han Zhang, Zhe Gan, Xiaolei Huang, and Xiaodong He. 2018. AttnGAN: Fine-Grained Text to Image Generation with Attentional Generative Adversarial Networks. In 2018 IEEE/CVF Conference on Computer Vision and Pattern Recognition. 1316-1324. https://doi.org/10.1109/ CVPR.2018.00143

[19] Han Zhang, Tao Xu, Hongsheng Li, Shaoting Zhang, Xiaogang Wang, Xiaolei Huang, and Dimitris N Metaxas. 2018. Stackgan++: Realistic image synthesis with stacked generative adversarial networks. IEEE transactions on pattern analysis and machine intelligence 41, 8 (2018), 1947-1962.

[20] Minfeng Zhu, Pingbo Pan, Wei Chen, and Yi Yang. 2019. Dm-gan: Dynamic memory generative adversarial networks for text-to-image synthesis. In Proceedings of the IEEE/CVF Conference on Computer Vision and Pattern Recognition. 5802-5810. 